International Journal of Pure and Applied Mathematics

Volume 88 No. 4 2013, 469-474

ISSN: 1311-8080 (printed version); ISSN: 1314-3395 (on-line version)

url: http://www.ijpam.eu

doi: http://dx.doi.org/10.12732/ijpam.v88i4.2

ijpam.eu

\title{
PARTITIONING OF LOOP-FREE SPERNER HYPERGRAPHS INTO TRANSVERSALS
}

\author{
R. Dharmarajan ${ }^{1}$, S. Palaniammal ${ }^{2}$ \\ ${ }^{1,2}$ Research and Development Centre \\ Bharathiar University \\ Coimbatore, INDIA \\ ${ }^{1}$ Department of Mathematics \\ SASTRA University \\ Thanjavur, Tamilnadu State, INDIA \\ 2 Department of Science and Humanities \\ Sri Krishna College of Technology \\ Coimbatore, INDIA
}

\begin{abstract}
This article explores possibilities of partitioning the vertex set of a given simple loop-free Sperner hypergraph into a union of transversals. Studies are done on the possible number of transversals in such partitions, followed by forming a hypergraph (on the vertex set of the given hypergraph) that consists of transversals for hyperedges.
\end{abstract}

AMS Subject Classification: 05C65

Key Words: hypergraph, hyperedge, Sperner, transversal, partition

\section{Introduction}

The set of all subsets (including the empty set $\phi$ ) of a nonempty set $V$ is denoted by $2^{V}$ and is called the power set [6] of $V$. The set $2^{V *}$ denotes the set of all nonempty subsets of $V$; that is, $2^{V *}=2^{V}-\{\phi\}$. A hypergraph [1] on a nonempty finite set $V$ is an ordered couple $H=(V, E)$ where $E$ is a family of nonempty subsets of $V$ such that $\bigcup_{X \in E} X=V$. The set $V$ is the vertex set of

Received: April 10, 2013

(c) 2013 Academic Publications, Ltd.

$\S$ Correspondence author url: www.acadpubl.eu 
$H$ and each member of $E$ is a hyperedge of $H$.

The cardinality of a finite set $V$ is denoted by $|V|$. A hyperedge $X$ in $\mathrm{H}$ with $|X|=1$ is a loop; more specifically, a hyperedge $X=\{a\}$ is a loop at the vertex $a$. A hypergraph is loop-free if $|X| \geq 2$ for every hyperedge $X$. If no hyperedge in $H$ equals all of $V$ then $H$ is non-trivial. If the members of $E$ are all distinct (that is, no two members coincide as subsets of $V$; or, $E \subseteq 2^{V *}$; or, $E$ has no repeated hyperedges) then $H$ is simple. If no member of $E$ is a subset (proper or otherwise) of another, then $H$ is a Sperner hypergraph. In some instances ([1] and [3]) Sperner hypergraphs and simple ones are deemed same, but there is a distinction [5] between the two: Sperner hypergraphs are simple but not conversely (1.1 of [2]).

A hypergraph $H=(V, E)$ is $r$-uniform (for some positive integer $r$ ) if $|X|$ $=r$ for every hyperedge $X$. Two vertices $x$ and $y$ are adjacent if $x \in X$ and $y \in X$ for some hyperedge $X$. $H$ is complete if every vertex is adjacent to every other vertex. $H$ is a partitioned hypergraph if the hyperedges form a partition [6] (or, a set partition) of $V$. A partitioned hypergraph is necessarily Sperner.

A Helly hypergraph is a Sperner hypergraph $H=(V, E)$, with $|V|>|E|$ $=k$, that has the following properties (with $E=\left\{X_{1}, \ldots, X_{k}\right\}$ ):

(i) The set $n(H)=\bigcap_{j=1 t o k} X_{j}$ is nonempty;

(ii) each $X_{j} \in E$ can be partitioned as $X_{j}=S_{j} \cup n(H)$ (that is, $S_{j} \cap n(H)=$ $\phi)$ with $S_{i} \cap S_{j}=\phi$ whenever $i \neq j$; in other words, the intersection of two distinct hyperedges is precisely $n(H)$.

In a Helly hypergraph, each $S_{j}$ as above is a cabal and the set $n(H)$ is the nucleus of the hypergraph.

This research work is a result of theoretical interest. Motivating ideas are (i) hypergraph colouring, treated in [1] in substantial detail, and (ii) bipartite graphs [4]. All the hypergraphs in this article are assumed non-trivial, loop-free, Sperner and having at least three vertices unless some unambiguous indication to the contrary is provided. If $H$ is a hypergraph, then $H=(V, E)$ unless another couple takes the place of $(V, E)$ explicitly.

\section{Partitioning by Transversals}

A transversal [1] in a hypergraph $H$ is a nonempty subset $T$ of the vertex set $V$ (i.e., $T \in 2^{V *}$ ) such that $T$ intersects each hyperedge in $H$ (i.e., $T \cap X \neq \phi$ for each hyperedge $X$ ).

A 2-transversal partition of $H$ is a partition $V=T_{1} \cup T_{2}$ such that $T_{1}$ and $T_{2}$ are transversals in $H$ (though it is not required that $T_{1}$ or $T_{2}$ be a hyperedge 
in $H$ ). If such a partition of $V$ exists then write $H \in 2-T P$; else $H \notin 2-T P$.

More generally, an $r$-transversal partition $(r \in N, r>1$, with $N$ being the set of positive integers) of $H$ is a partition $V=T_{1} \cup \ldots \cup T_{r}$ such that each $T_{j}$ $(j=1$ through $r)$ is a transversal in $H$. Write $H \in r-T P$ if such a partition of $V$ exists; else $H \notin r-T P$. If $H \in r-T P$ with a partitioning $V=T_{1} \cup \ldots \cup T_{r}$ by transversals, then each $T_{j}$ is a component transversal.

Let $\tau^{*}(H)=\{r \in N \mid H \in r-T P\}$. Then $\tau^{*}(H)$ is a finite subset of $N$, $1 \notin \tau^{*}(H)$, and $\tau^{*}(H)$ may be empty. If $\tau^{*}(H) \neq \phi$ then let $\Omega(H)=\max$ $\tau^{*}(H)$; and if $\tau^{*}(H)=\phi$, then set $\Omega(H)=0 . \Omega(H)$ is the transversal partition number of $H$. Clearly $\Omega(H) \geq 2$ if $\tau^{*}(H) \neq \phi$.

Proposition 2.1. If $T_{1}$ and $T_{2}$ are transversals in $H$ then so is $T_{1} \cup T_{2}$. (The proof is elementary.)

Proposition 2.2. If $H \in r-T P$ and if $r \geq 3$ then $H \in k-T P$ for each $k$ with $2 \leq k<r$.

Proof. Let $V=T_{1} \cup \ldots \cup T_{r}$ be a partition of $V$ such that each $T_{j}(j$ $=1$ through $r$ ) is a transversal in $H$. Then $V=T_{1} \cup \ldots \cup T_{r-2} \cup M$, where $M=T_{r-1} \cup T_{r}$, is clearly a partition of $V$ by $r-1$ transversals, whence $H \in(r-$ 1) $-T P$, from which the conclusion follows for any $k \in N$ with $2 \leq k<r$.

Proposition 2.3. Let $r \in N, r \geq 2$. If $H \in r-T P$ then $|X| \geq r$ for each hyperedge $X$.

The proof of 2.3 is straightforward. The converse is not true in the general case, as 2.4 shows. But the converse does hold for all partitioned hypergraphs (2.5) and all Helly hypergraphs (2.6), so long as these are loop-free.

Example 2.4. Let $H_{n, r}(n>r \geq 2)$ denote the complete $r$-uniform hypergraph on a finite set $V$ where $n=|V|$; that is, the hyperedges of $H_{n, r}$ are precisely all the subsets $X$ of $V$ satisfying $|X|=r$. If $T$ is any proper transversal in $H_{n, 2}$ then $T$ has at least two elements. Let $x, y \in T$. Then $V-T$ does not meet the hyperedge $\{x, y\}$. Consequently $H_{n, 2} \notin 2-T P$ though $|X|=2$ for each hyperedge $X$.

Proposition 2.5. Let $r \in N, r \geq 2$ and $H=(V, E)$ be a partitioned hypergraph. Then $H \in r-T P$ if and only if $|X| \geq r$ for each hyperedge $X$. Moreover, $\tau^{*}(H)=\min |X|$ as $X$ runs over $E$.

Proof. ( $\Longrightarrow$ ) follows from 2.3 .

$(\Longleftarrow)$ Since $H$ is partitioned, $|X| \geq r$ ensures the presence of $r$ pair wise disjoint transversals whose union is $V$. The last part, namely $\tau^{*}(H)=\min |X|$ as $X$ runs over $E$, is then evident, 
Proposition 2.6. Let $r \in N, r \geq 2$ and $H=(V, E)$ be a Helly hypergraph. Then $H \in r-T P$ if and only if $|X| \geq r$ for each hyperedge $X$. Moreover, $\tau^{*}(H)=\min |X|$ as $X$ runs over $E$. xms

Proof. $(\Longrightarrow)$ follows from 2.3 .

$(\Longleftarrow)$ Write $E=\left\{X_{1}, \ldots, X_{k}\right\}$ and let $n(H)=\left\{z_{1}, \ldots, z_{q}\right\}$. Set $T_{j}=\left\{z_{j}\right\}$ for $j=1$ through $q$. Then each $T_{j}$ is a transversal in $H$. Further, $r>q$, and so let $p=r-q$. Writing $X_{j}=S_{j} \cup n(H)$ (with $S_{j} \cap n(H)=\phi$ and $S_{i} \cap S_{j}=\phi$ whenever $i \neq j$ ) for $j=1$ through $k$, we at once have $\left|S_{j}\right| \geq p$ for each $j$, using which we come up with $p$ transversals - say $U_{1}$ through $U_{p}$ - that are pair wise disjoint and that cover $V-n(H)$. Then, the $q+p(=r)$ transversals $T_{1}, \ldots, T_{q}, U_{1}, \ldots, U_{p}$ are pair wise disjoint and their set union is $V$, whence $H \in r-T P$. The part about $\tau^{*}(H)$ is then straightforward.

Proposition 2.7. If $H \in r-T P$, then $|T \cap X|<|X|$ for each component transversal $T$ and for every hyperedge $X$.

Proof. Let $V=T_{1} \cup \ldots \cup T_{r}$ be a transversal partition of $H$. Were it to happen $\left|T_{j} \cap X\right|=|X|$ for some hyperedge $X$ and some component $T_{j}$ then it would lead to $X \subseteq T_{j}$, a contradiction.

For an arbitrary loop-free Sperner hypergraph, is there a necessary and sufficient condition for $H \in r-T P$ for a given positive integer $r \geq 2$ ? For $r=2$, there is one (2.8) but then it might not be easy to implement (in an algorithm) when the number of hyperedges is very large (not to speak of the number of vertices). Moreover, 2.8 cannot be extended to $r>2$, as 2.9 shows.

Proposition 2.8. $H \in 2-T P$ if and only if (i) $|X| \geq 2$ for each hyperedge $X$ and (ii) there is a transversal $T$ in $H$ such that $|T \cap X|<|X|$ for every hyperedge $X$.

Proof. $(\Longrightarrow)$ If $H \in 2-T P$, then (i) holds by 2.3 and (ii) by 2.7 .

$(\Longleftarrow)$ Assume (i) and (ii) hold. Let $T$ be a transversal in $H$ with $|T \cap X|<\mid$ $X \mid$ for every hyperedge $X$. Then $T$ is a proper subset of $V$. Let $V-T=W$. Were $W \cap Y=\phi$ for some hyperedge $Y$, then $T \cap Y=Y$, running contrary to $|T \cap Y|<|Y|$. So $W$ is a transversal in $H$, whence $H \in 2-T P$.

Example 2.9. Let $V=\{1,2,3,4\}$ and let $H_{4,3}$ denote the complete 3 uniform hypergraph on $V . Y=\{1,2\}$ is a transversal such that $|Y \cap X|<|X|$ for every hyperedge $X$. If $T$ is a transversal in $H_{4,3}$ then $|T| \geq 2$. But this rules out the intersection, for instance, of the hyperedge $\{1,2,3\}$ with more than two disjoint transversals. Consequently $H_{4,3} \notin 3-T P$. Still, $H_{4,3} \in 2-T P$ because $\{1,2\}$ and $\{3,4\}$ are transversals in $H_{4,3}$ with $V=\{1,2\} \cup\{3,4\}$. 


\section{Transversal Conjugates}

Let $H_{1}$ and $H_{2}$ be two hypergraphs on the same set $V$. If each hyperedge in $H_{1}$ is a transversal in $H_{2}$ then $H_{1}$ is a transversal conjugate of $H_{2}$, written $H_{1}(=\tau) H_{2}$; else $H_{1}(\neq \tau) H_{2}$.

Proposition 3.1. Let $H_{1}, H_{2}$ and $H$ be hypergraphs on the same set $V$.

(i) If $H_{1}(=\tau) H_{2}$ then $H_{2}(=\tau) H_{1}$; for this reason, $H_{1}$ and $H_{2}$ are also called transversal conjugates.

(ii) $H(=\tau) H$ if and only if the hyperedges of $H$ are pair wise intersecting.

Example 3.2. If $H_{1}(=\tau) H_{2}$ and $H_{2}(=\tau) H_{3}$ then it is not necessary that $H_{1}(=\tau) H_{3}$. Consider the hypergraphs $H_{1}=\left(V, E_{1}\right), H_{2}=\left(V, E_{2}\right)$ and $H_{3}=\left(V, E_{3}\right)$ on $V=\{a, b, c, d\}$, where: $X_{1}=\{a, b\}, X_{2}=\{c, d\}, X_{3}=\{a, d\}$, $X_{4}=\{b, c\}, X_{5}=\{a, b, c\}$ and $X_{6}=\{c, d\} ; E_{1}=\left\{X_{1}, X_{2}\right\}, E_{2}=\left\{X_{3}, X_{4}\right\}$ and $E_{3}=\left\{X_{5}, X_{6}\right\}$. Then $H_{1}(=\tau) H_{2}$ and $H_{2}(=\tau) H_{3}$ but $H_{1}(\neq \tau) H_{3}$.

Proposition 3.3. Let $H=(V, E)$ with $E=\left\{X_{1}, \ldots, X_{k}\right\}$. Let $V=$ $T_{1} \cup \ldots \cup T_{r}$ be an r-transversal partition of $V$ and let $E_{T(r)}=\left\{T_{1}, \ldots, T_{r}\right\}$. Then $H_{T(r)}=\left(V, E_{T(r)}\right)$ is a hypergraph on $V$. Also, $H(=\tau) H_{T(r)}$.

The proofs of (3.1) and (3.3) are straightforward.

\section{Resume}

Partitioning a loop-free Sperner hypergraph into two transversals is relatively simpler than the general case where the number of transversals required is arbitrary. The authors are working on the latter case, especially for a large number of vertices and hyperedges. This kind of partitioning could have close associations with hypergraph colouring.

\section{Acknowledgments}

The authors express their thanks to (1) Professor R. Sethuraman, Vice Chancellor, SASTRA University and (2) D. Ramachandran, Director, Abel-Jacobi Research Foundation, Palghat (Kerala State, India) for their unstinted encouragement and support. 


\section{References}

[1] C. Berge, Hypergraphs-Combinatorics of finite sets, North-Holland Mathematical Library, Holland (1989).

[2] R. Dharmarajan, D. Ramachandran, On connections between dominating sets and transversals in simple hypergraphs, International Journal of Pure and Applied Mathematics, 80, No. 5 (2012), 21-26.

[3] T. Eiter, G. Gottlob, Identifying the minimal transversals of a hypergraph and related problems, SIAM Journal on Computing, 24, No. 6 (1995), 1278-1304, doi: 10.1137/S0097539793250299.

[4] John M. Harris, Jeffry L. Hirst, Michael J. Mossinghoff, Combinatorics and Graph Theory, Springer, USA (2008), doi: 10.1007/978-0-387-79711-3.

[5] K.H. Rosen, Handbook of Discrete and Combinatorial Mathematics, CRC Press LLC, USA (2000).

[6] R.R. Stoll, Set Theory and Logic, Dover, USA (1963). 